\title{
Inhaltsverzeichnis \\ (Table of Contents)
}

\section{A. Einführung \\ (Introduction)}

1. Die englische Rechtschreibung (Orthography) . . . . . 7

2. Die englische Aussprache (Pronunciation) . . . . . . . 8

3. Das englische Alphabet (Alphabet) . . . . . . . . . . 11

4. Der englische Tonfall (Intonation) . . . . . . . . . . . 11

5. Der englische ebene Nachdruck (Level-stress). . . . . . 1'1

6. Die englischen Stark- und Schwachdruckformen (Stressed and unstressed words and syllables) . . . . . . . . . 15

\section{B. Sprachlehre \\ (Grammar)}

1. Geschlechtswort (Article)

$\S 1$. Bestimmtes Geschlechtswort (Definite article) . . . . . 18

$\S 2$. Unbestimmtes Geschlechtswort (Indefinite article) . . . 18

2. Hauptwort (Noun)

$\S 3$. Geschlecht (Gender) . . . . . . . . . . . . . . . 19

§. Mehrzahl (Plural) . . . . . . . . . . . . . . . . 21

$\S \S 5-8$. Formen und Gebrauch der Fälle (Forms and use of the cases $)$. . . . . . . . . . . . . . . . . 22

3. Eigenschaftswort (Adjective)

§9. Formen und Gebrauch (Forms and use) . . . . . . . . 25

$\S \S 10-11$. Steigerung (Degrees of comparison) . . . . . . . 26

4. Umstandswort (Adverb)

$\S 12$. Formen und Gebrauch (Forms and use) . . . . . . 28

5. Zahlwort (Numeral)

$\S 13$. Grundzahlen (Cardinal numbers) . . . . . . . . . . 30

$\S 14$. Ordnungszahlen (Ordinal numbers) . . . . . . . . . . . 31

§ 15. Andere Zahlformen (Other numerals) . . . . . . . . . . 32 


\section{Fürwort (Pronoun)}

$\S \S 16-18$. Persönliches Fürwort (Personal pronoun) . . . . . 32

§19. Zurückbezügliches Fürwort (Reflexive pronoun) . . 34

$\S 20$. Besitzanzeigendes Fürwort (Possessive pronoun). . . 35

$\S 21$. Hinweisendes Fürwort (Demonstrative pronoun) . . . 35

$\S 22$. Bezügliches Fürwort (Relative pronoun) . . . . . . 35

$\S 23$. Bestimmendes Fürwort (Determinative pronoun) . 36

$\S 24$. Fragendes Fürwort (Interrogative pronoun) . . . . 36

$\S 25$. Unbestimmtes Fürwort (Indefinite pronoun) . . . . 37

7. Verbältniswort (Preposition)

$\S 26$. Formen und Gebrauch von 50 üblichen Verhältniswörtern in alphabetischer Reihenfolge (Forms and use of 50 common prepositions in alphabetical order) . . . . 38

\section{Zeitwort (Verb)}

$\S \S 27-28$. Vollständige Hilfszeitwörter (Complete auxiliary verbs) . . . . . . . . . . . . . . . 50

§29. Unvollständige Hilfszeitwörter (Defective auxiliary verbs) . . . . . . . . . . . . . . . 52

$\S 30$. Vollzeitwörter (Principal verbs) . . . . . . . . . . . 53

$\S 31$. Darstellung der Biegung eines schwachen und eines starken Zeitworts (Tabular view of the conjugation of a weak and a strong verb) . . . . . . . . . . . . . . 56

§32. Liste der unregelmäßigen schwachen Zeitwörter (List of the irregular weak verbs) . . . . . . . . . . . . . . 58

$\S 33$. Liste der starken Zeitwörter (List of the strong verbs) $\quad$. 60

$\S 34$. Liste der Zeitwörter mit gemischten schwachen und starken Formen (List of the verbs with mixed weak and strong forms) . . . . . . . . . . . . . . . . . . 62

$\S 35$. Gebrauch der Zeiten (Use of the tenses) . . . . . . 62

$\S 36$. Wirklichkeitsform und Möglichkeitsform (Indicative mood and subjunctive mood) . . . . . . . . . . . . 64

$\S 37$. Nennform (Infinitive) und abgewandelte Nennform (Gerund)

$\S 38$. Mittelwörter (Participles) . . . . . . . . . . . . 66

9. Bindewort (Conjunction)

$\S 39$. Nebenordnung (Parataxis or co-ordination) . . . . . 67

$\S 40$. Unterordnung (Hypotaxis or sub-ordination) . . . . 67 
10. Wortstellung (Word-order)

$\S 41$. Funktion der Wortstellung (Function of the word-order) 69 $\S 42$. Regelmäßige Wortstellung (Regular word-order) . . . 69 §43. Umstellung (Inverted order) . . . . . . . . . . . . 70

\section{Gesprächsbeispiele \\ (Everyday Talk in Spoken English)}

1. Grußwechsel (Greetings) . . . . . . . . . . . . . . 72

2. Vorstellung (Introductions) . . . . . . . . . . . . . . 72

3. Danksagung (Thanksgivings) . . . . . . . . . . . . 73

4. Entschuldigung (Apologies) . . . . . . . . . . . 73

5. Wegerkundung (Asking the way) . . . . . . . . 74

6. Verabredung (Appointments) . . . . . . . . . . . . 75

7. Erste Unterhaltung auf Englisch (First conversation in English) . . . . . . . . . . . . . . . . . 75

8. Zerstreuung (Entertainments) . . . . . . . . . . . . . 76

9. Das Wetter (Weather) . . . . . . . . . . . . . . 78

10. Im Geschäft (Shops and shopping) . . . . . . . . . . 79

11. Das Reisen (Travelling) . . . . . . . . . . . . . . . 81

12. Zu Hause (At home). . . . . . . . . . . . . . . . . . 83

Sachverzeichnis (Subject index) . . . . . . . . 86 
,Wer fremde Sprachen nicht kennt, weiß nichts von seiner eigenen." (GOETHE) 